University of Nebraska - Lincoln

DigitalCommons@University of Nebraska - Lincoln

Robert Katz Publications

Research Papers in Physics and Astronomy

November 1986

\title{
The Fricke Dosimeter as a 1-Hit Detector
}

Robert Katz

University of Nebraska-Lincoln, rkatz2@unl.edu

G. L. Sinclair

University of Nebraska-Lincoln

M. P. R. Waligorski

Institute of Nuclear Physics, Krakow, Poland

Follow this and additional works at: https://digitalcommons.unl.edu/physicskatz

Part of the Physics Commons

Katz, Robert; Sinclair, G. L.; and Waligorski, M. P. R., "The Fricke Dosimeter as a 1-Hit Detector" (1986).

Robert Katz Publications. 91.

https://digitalcommons.unl.edu/physicskatz/91

This Article is brought to you for free and open access by the Research Papers in Physics and Astronomy at DigitalCommons@University of Nebraska - Lincoln. It has been accepted for inclusion in Robert Katz Publications by an authorized administrator of DigitalCommons@University of Nebraska - Lincoln. 
Published in Nuclear Tracks and Radiation Measurements 11:6 (1986), pp. 301-307; now known as Radiation Measurements; formerly International Journal of Radiation Applications and Instrumentation. Part D. Nuclear Tracks and Radiation Measurements. Published by Pergamon Journals Ltd. Used by permission. http://www.sciencedirect.com/science/journal/13504487

Submitted September 12, 1986; revised November 25, 1986.

\title{
The Fricke Dosimeter as a 1-Hit Detector
}

\author{
R. Katz, ${ }^{1}$ G. L. Sinclair, ${ }^{1}$ and M. P. R. Waligorski ${ }^{2}$ \\ ${ }^{1}$ University of Nebraska-Lincoln, Lincoln, Nebraska 68588-0111, USA \\ ${ }^{2}$ Institute of Nuclear Physics, Krakow, Poland
}

\begin{abstract}
A fit to the experimental data for the response of the Fricke dosimeter to energetic heavy ions is obtained using a calculation of the relative effectiveness of a 1-hit detector, from track theory. We use 2 fitted parameters, the target size, $a_{0}$, which may be thought to represent a "diffusion length," and $E_{0}$, the dose of gamma-rays at which there is an average of one hit per target (the D-37 dose), and a new algorithm for the average radial distribution of dose in liquid water from the passing ion. The $G$ value for ions is then given as the product of the calculated relative effectiveness and the experimental $G$ value for gamma rays.
\end{abstract}

\section{Introduction}

Two very different types of explanations have been offered for the variation in yield of the ferrous sulphate (Fricke) dosimeter after bombardment with energetic heavy ions of different stopping power. One of these, based on diffusion kinetics, is widely used in radiation chemistry. The other is based upon track theory, and originates with a model for the relative biological effectiveness of dry enzymes and viruses (Butts, 1967), later extended to other detectors (Katz, 1972).

As applied to the Fricke dosimeter (Chatterjee, 1980) the diffusion kinetic model uses a rather extensive array of chemical reactions, reaction rates, diffusion constants, and a set of linear differential equations through which it proceeds from initially formed radicals to the final differential yield. It makes use of an imaginative model of the energy deposition about an ion's path, including such terms as spurs, blobs, short tracks, penumbra, and core, the latter being one of its more vulnerable aspects. Neither measurements nor Monte Carlo calculations of the radial dose distribution offer any hint of the track core in energy deposition specified in the diffusion kinetic model.

As in earlier work, we make use of the model of the 1-hit detector and show that a calculation of the relative effectiveness of a 1-hit detector matches the response of the Fricke dosimeter to energetic heavy ions. Here we exploit a new formulation of the radial distribution of dose (Waligorski, 1986) to advantage. We have been able to find detector parameters such that the calculated relative yields agree with measured yields for the
Fricke dosimeter at particle energies above $1 \mathrm{MeV} / \mathrm{u}$. At lower energies there is some disagreement between our calculations and experiment which we attribute to uncertainties in our knowledge of the radial distribution of dose.

In this model all complexities of diffusion kinetics are avoided, but at the price of loss of detailed knowledge of the dynamics of the chemical process. A new insight is gained, namely that diffusion kinetics repetitively calculates the response of the Fricke dosimeter to the tangle of electrons in the penumbra, which is not appreciably different than calculating the response to different doses of gamma rays or energetic electrons.

In the track theory model, an exponential response of the Fricke dosimeter to gamma-rays is assumed, and described by a characteristics parameter $E_{0}$ at which there is an average of 1 hit per sensitive target. It is assumed that the target is a sphere of water of radius $a_{0}$ surrounding a $\mathrm{Fe}^{2+}$ ion such that a hit within the sphere can initiate the array of events which diffuse to the $\mathrm{Fe}^{2+}$ ion and interact with it, transforming it to an $\mathrm{Fe}^{3+}$ ion. The probability for this event is calculated from the average energy deposited in the target volume, the local dose, as a function of the radial distance from the ion's path. Integration of the radial probability function yields an action cross section, and subsequently the relative effectiveness.

\section{The Track Model of a 1-Hit Detector}

Following the prescription of biological target theory we take the response of a 1-or-more hit detector to obey 
the cumulative Poisson distribution. Thus the probability $P$ that an "action" occurs in a sensitive element experiencing a dose $E$ is

$$
P(D)=1-\exp \left(-E / E_{0}\right)
$$

where $E_{0}$ is the dose at which there is an average of 1 hit per target. Because of fluctuations in the energy deposition, some $63 \%$ of the targets are hit at this dose. In radiobiology $E_{0}$ is also known as the D-37 dose for gammarays, for $1 / e$ or $37 \%$ of the irradiated targets of a 1-hit detector survive at this dose level. For the present calculation $E_{0}$ is treated as a fitted parameter, for the dose response curve of the Fricke dosimeter at very high doses has not been measured. It is frequently assumed that the yield of the Fricke dosimeter is independent of dose, but this is only true at low doses. Measurement with pulse of electrons (Sehested, 1969; Hart, 1963) have shown there to be a decline in yield with increasing dose.

In track physics it is assumed that the response of equation (1) to (the secondary electrons from) gammaray irradiation is applicable to the dose deposited by delta-rays about an ion's path. It is here assumed that we are calculating the average response of sensitive elements in equivalent coaxial shells at the same radial distance from the paths of many ions. Since the radial dose falls off rapidly, approximately inversely with the square of the distance from an ion's path, we take it that the average dose in a sensitive element, of radius $a_{0}$, is descriptive of the response of the entire element whose center is located at radial distance $t$ from the ion's path. Thus the average dose is calculated and substituted in equation (1) to find the probability $P(t)$ for activation of such elements.

From $P$ we can calculate the action cross-sections for this interaction. Here we imagine that a single ion is passing down a channel $1 \mathrm{~cm}^{2}$ in area, and that it may interact with a single target somewhere in that channel. The objective probability that it may take place is given as the fraction of successes in a large number of repeated trials (an average value). The probability is then stated as the ratio of the action cross-section or to the cross sectional area of the channel. The cross-section is calculated as

$$
\sigma=2 \pi \int_{t=0}^{t=T} P(t) \mathrm{d} t
$$

where $T$ is the maximal radial penetration of delta-rays.

We take the radiosensitivity, $k$, of the detector to be the reciprocal of the dose at which there is $37 \%$ survival, so for $\gamma$-rays we have

$$
k_{r}=1 / E_{0}
$$

For heavy ions irradiating thin specimens (track segment irradiation) the radiosensitivity is

$$
k_{i}=\sigma / L
$$

where $L$ is the stopping power (Linear Energy Transfer $=\mathrm{LET}$ ) of the ion. Then we define the relative efficiency to be

$$
R E(\text { calculated })=k_{i} / k_{\gamma}=\sigma E_{0} / L
$$

In the case of the Fricke dosimeter the experimental value of the relative efficiency is the ratio of the $G$ value for ion bombardment to the $G$ value for gamma-ray irradiation, so that

$$
R E \text { (measured })=G_{i}\left(\mathrm{Fe}^{3+}\right) / G_{r}\left(\mathrm{Fe}^{3+}\right) .
$$

Thus we compare the calculation of equation (5) to the measurement of equation (6), after searching for parameters which yield the best agreement between the two for all available data, particularly for ion bombardment with particles of energy above $1 \mathrm{MeV} / \mathrm{u}$.

To calculate the response of thick detectors to stopping ions, of initial kinetic energy $T$, and having range $R$, we integrate the response over the path length. In our expression for relative effectiveness, equation (5), we must then replace $\sigma$ and $L$ by their average values defined through the expressions

$$
\sigma_{\text {ave }} R=\int_{\mathrm{r}=0}^{\mathrm{r}=R} \sigma \mathrm{d} r=\int_{T_{i}}^{0} \sigma / L \mathrm{~d} T
$$

and

$$
L_{\text {ave }}=T_{i} / R \text {. }
$$

When calculating the $G$ values for stopping particles, we note that the fractional error from the stopping end of the track due to uncertainties in the radial dose distribution at particle energies below $1 \mathrm{MeV} / \mathrm{u}$ will loom large for particles of low initial energy, but will be somewhat diminished for high energy particles in proportion to their greater range.

\begin{tabular}{|c|c|c|c|c|}
\hline Ion & $\begin{array}{c}E \\
(\mathrm{MeV} / \mathrm{u})\end{array}$ & $\begin{array}{c}G \\
(\exp )\end{array}$ & $\begin{array}{c}G \\
\text { (model) }\end{array}$ & $\begin{array}{r}(G(\text { model }) / \\
G(\exp )-1) \times 100\end{array}$ \\
\hline C & $\begin{array}{r}60 \\
100 \\
200 \\
300\end{array}$ & $\begin{array}{l}10.14 \\
10.93 \\
12.03 \\
12.53\end{array}$ & $\begin{array}{l}10.36 \\
11.49 \\
12.87 \\
13.50\end{array}$ & $\begin{array}{l}+2.00 \\
+5.18 \\
+6.98 \\
+7.81\end{array}$ \\
\hline $\mathrm{Ne}$ & $\begin{array}{r}80 \\
100 \\
200 \\
300\end{array}$ & $\begin{array}{r}9.28 \\
9.68 \\
10.82 \\
11.12\end{array}$ & $\begin{array}{c}8.99 \\
9.469 \\
11.00 \\
11.84\end{array}$ & $\begin{array}{l}-3.0 \\
-2.17 \\
+1.2 \\
+6.47\end{array}$ \\
\hline $\mathrm{Ar}$ & $\begin{array}{l}100 \\
200 \\
300\end{array}$ & $\begin{array}{l}8.05 \\
9.00 \\
9.60\end{array}$ & $\begin{array}{l}7.665 \\
8.817 \\
9.554\end{array}$ & $\begin{array}{l}-5.0 \\
-2.07 \\
-0.48\end{array}$ \\
\hline
\end{tabular}

\section{Results}

By comparison of our calculations with the experimental results (Christman, 1981) arising from the irradiation of ferrous sulfate solutions with $\mathrm{C}, \mathrm{Ne}$, and Ar ions

Table 1. Differentiation yields, from track segment irradiation, of the aerated ferrous sulfate solution. Data from Christman et al. (1981). Calculations are from present model. 


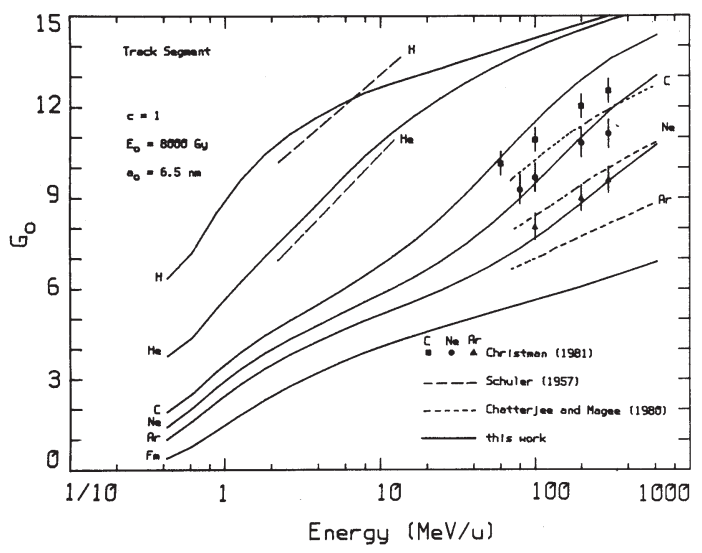

Figure 1. Calculated values, heavy lines this model, of $G_{0}$, (low dose) values for track segment irradiation of the Fricke dosimeter with $\mathrm{H}, \mathrm{He}, \mathrm{C}, \mathrm{Ne}, \mathrm{Ar}, \mathrm{Fm}$. Data points from Christman (1981) dashed lines for C, Ne, Ar are calculated values from Chatterjee and Magee (1980) while dashed lines for $\mathrm{H}$ and $\mathrm{He}$ are track segment values extracted by Chatterjee and Magee from stopping particle data of Schuler and Allen (1957).

of energy ranging from 60 to $300 \mathrm{MeV} / \mathrm{u}$, radiosensitivity parameters $a_{0}=6.5 \mathrm{~nm}$ and $E_{0}=8000$ Gy were extracted. As discussed earlier, the relative effectiveness of a 1-hit detector was calculated, and this was multiplied by a reference value of 15.6 to find the value of $G_{i}$ for the ion bombardment. As shown in Table 1, our calculated results were then within $8 \%$ of the experimental values for $\mathrm{C}$ bombardment, within $7 \%$ for $\mathrm{Ne}$, and within $5 \%$ for Ar. These fitted values may be compared to the discrepancies found by these authors of about $6 \%$ for $\mathrm{C}$ and
$13 \%$ for Ar between their diffusion kinetic model calculations and experiment.

In Figure 1 we show our calculated $G_{0}$ (low dose) values for track segment irradiation with a range of ions and ion energies as heavy lines. Shown as dashed lines for $\mathrm{H}$ and $\mathrm{He}$ are values obtained from the stopping particle work of Schuler and Allen (1957) by Chatterjee and Magee (1980). Dashed lines for C, Ne, and Ar are calculated values, also from Chatterjee and Magee. The data points are from Christman (1981).

Using these parameters, we then calculated the yield for stopping $\mathrm{D}, \mathrm{He}$, and $\mathrm{C}$ ions of maximum initial energy $10 \mathrm{MeV} / \mathrm{u}$ for comparison with data (Schuler, 1957, 1967) and display these results in Figure 2. We use the notation $G_{0}$ in this figure and elsewhere to represent the $G$ value at low doses of the bombarding particles.

Calculations made for comparison with the data obtained for irradiations with stopping protons, deuterons, and alpha particles of low energy (Hart, 1956; Gordon, 1961) yielded results shown in Figure 3. As expected the largest deviations are found for alpha particles of the lowest energy.

A comparison of our stopping particle calculation with some recent measurements with a series of ions from He to $\mathrm{C}$ at $20 \mathrm{MeV}$ is shown in Table 2 (LaVerne, 1983). The discrepancy between calculated and experimental values exceeds $10 \%$ for $\mathrm{Be}, \mathrm{B}$, and $\mathrm{C}$ irradiations reaching a maximum of about $21 \%$ for $C$, of initial energy $1.7 \mathrm{MeV} / \mathrm{u}$. In part these discrepancies must be attributed to uncertainties in our knowledge of the "effective charge," the radial dose distribution, and the

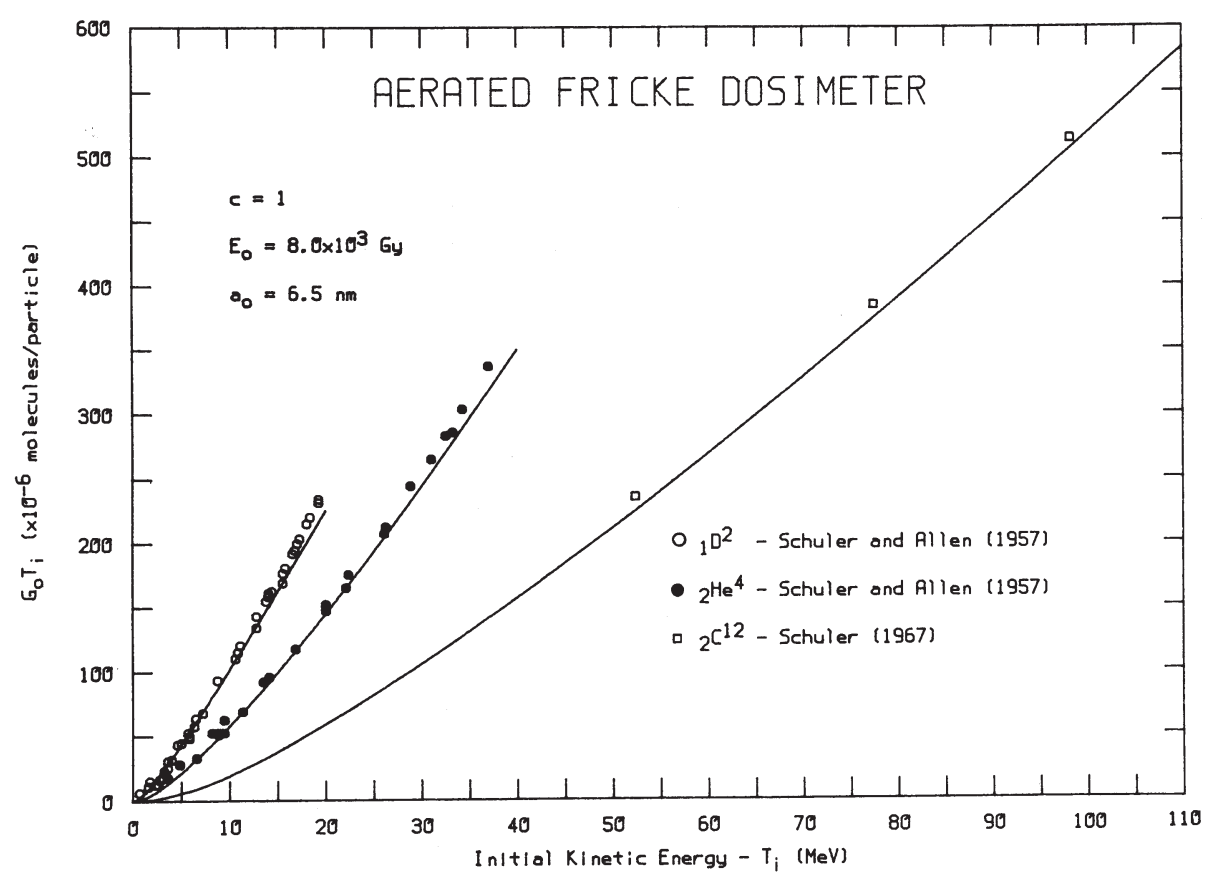

Figure 2. The product of the measured $G$ value, represented here as $G_{0}$, by the initial kinetic energy $T_{i}$ of the particles of the irradiating beam is plotted against the initial kinetic energy. Data points are from Schuler (1967) and Schuler and Allen (1957), while the lines are calculated from the present model. 


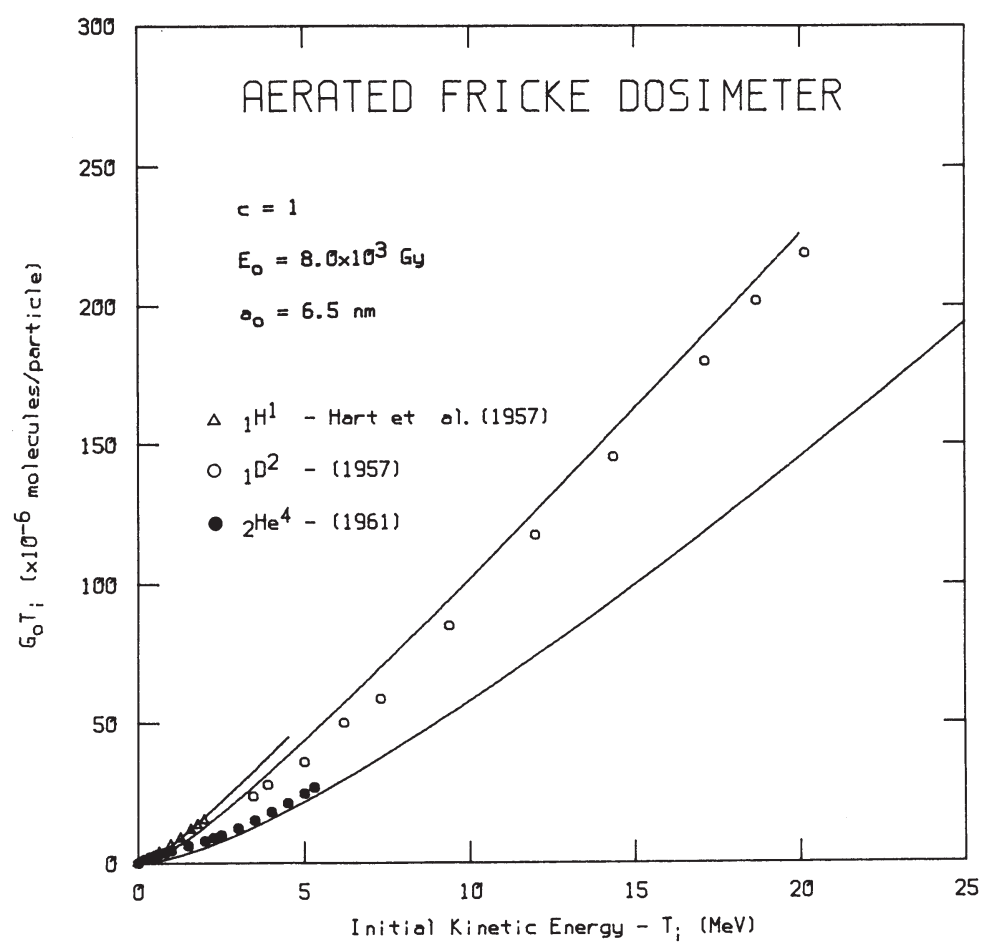

Figure 3. See caption of Figure 2, except that data points are from Gordon and Hart (1961) and Hart, Ramler and Rocklin (1956).

stopping power at these low energies. We use a table of Janni for the stopping power of protons in water multiplied by the square of the effective charge for heavy ions (see Appendix) (Janni, 1982).

\section{Discussion}

Our earlier calculation of the response of the Fricke dosimeter (Katz, 1972) yielded similar results but with somewhat different parameters. There we found $a_{0}=$ $6 \mathrm{~nm}$ and $E_{0}=5000 \mathrm{~Gy}$. These earlier parameters and the radial dose distribution then used yield calculations which differ by $12-23 \%$ from the measurements of Christman et al. (1981). Though the overall qualitative results are similar in the present calculation as earlier, the quantitative agreement with experimental findings is now somewhat better.

In justification of the numerical values of our present fitted parameters, we calculate the "energy deposited" in a sphere of radius $6.5 \mathrm{~nm}$ by a dose of $8000 \mathrm{~Gy}$, and

Table 2. Integral yields, from irradiation with stopping particles, of aerated ferrous sulfate solution. Data from La Verne and Schuler (1983). Calculations are from the present model.

\begin{tabular}{lcccc}
\hline Ion & $\begin{array}{c}E \\
(\mathrm{MeV} / \mathrm{u})\end{array}$ & $\begin{array}{c}G \\
(\exp )\end{array}$ & $\begin{array}{c}G \\
(\text { model })\end{array}$ & $\begin{array}{c}(G(\text { model }) / \\
\text { (exp) }-1) \times 100\end{array}$ \\
\hline $\mathrm{He}$ & 5.0 & 7.83 & 7.26 & -7.18 \\
$\mathrm{Li}$ & 3.85 & 5.34 & 5.432 & +1.37 \\
$\mathrm{Be}$ & 2.22 & 4.56 & 3.916 & -14.14 \\
$\mathrm{~B}$ & 1.818 & 3.95 & 3.315 & -16.08 \\
$\mathrm{C}$ & 1.666 & 3.73 & 2.933 & -21.37 \\
\hline
\end{tabular}

find it to be $58 \mathrm{eV}$. This is consistent with the value of 60 $\mathrm{eV}$ customarily assumed for the production of a "hit" in an enzyme molecule (Dertinger, 1970) through which a "target molecular weight" is calculated.

There are independent justifications for both $a_{0}$ and $E_{0}$. In a study of the effect of ferrous sulphate concentration on the yield of oxidation of the ferrous ion from recoil radiations from the absorption of neutrons in boron, it was found (Schuler, 1958) that the yield approached "saturation" at a $1 \mathrm{mM}$ concentration of $\mathrm{Fe}^{2+}$ ions. We interpret this finding as implying the existence of a diffusion length which limits the "reach" of the products of the initial ionization event in water. A $1 \mathrm{mM}$ concentration implies a mean separation of $11.8 \mathrm{~nm}$ for $\mathrm{Fe}^{2+}$ ions, or a "target" radius of $6 \mathrm{~nm}$ as compared to our fitted value of $a_{0}$ of $6.5 \mathrm{~nm}$.

Hart (1963) found a decline in yield with an increase in dose per pulse from electron accelerators delivering pulses of order 100 krads per pulse in periods of microseconds. At a dose of 290 krads per pulse he found a $G$ value of $11.3 \pm 0.5$. Our model of the 1-hit detector implies that there will be an exponential decline in yield with dose $D$ of the form

$$
G=G_{0} \mathrm{e}^{-D / E_{0}}
$$

which yields a value of $G$ at 290 krads of 10.9, within the range of the Hart measurement.

It is interesting to compare projections of the response of the Fricke dosimeter from the 1-hit model with those made by Chatterjee and Magee from their diffusion kinetic model, for both models compare to the same sets 


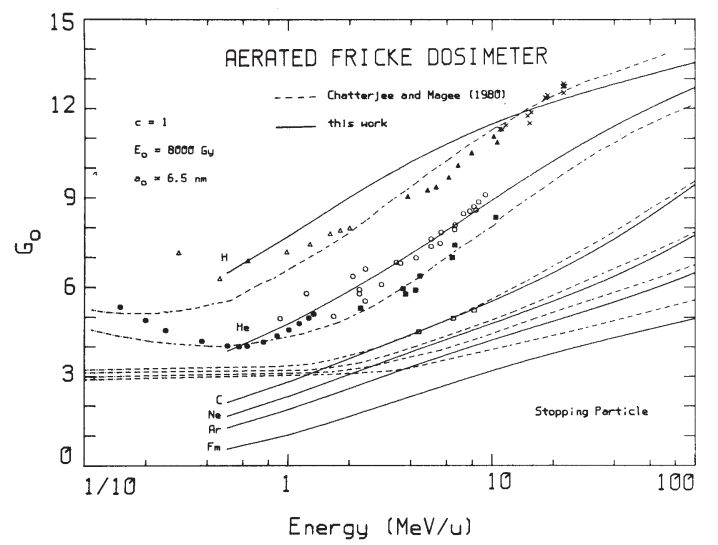

Figure 4. Calculated values of the $G$ value (here called $G_{0}$ ) of the aerated Fricke dosimeter for stopping particle irradiation are plotted against particle energy, for ions $\mathrm{H}, \mathrm{He}, \mathrm{C}, \mathrm{Ne}, \mathrm{Ar}$, and Fm. Values from the present model (solid lines) are compared to the results of Chatterjee and Magee (1980) (dashed lines). At energies above $1-2 \mathrm{MeV} / \mathrm{n}$, the curves are similar in character but differ quantitatively, though the differences are limited since both models seek to fit the same set of data. At low ion velocities we lack information about the radial dose distribution and stopping power to make calculations from the present model. Various experimental results are also shown.

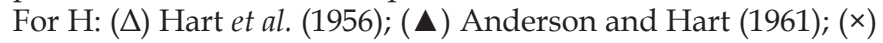
Kochanny et al. (1963). For He: (०) Schuler and Allen (1956); (•) Gordon and Hart (1961); (•) Anderson and Hart (1961). For C: (口) Schuler (1967).

of data for their ultimate justification. In Figure 4 we display the projections of the differential yield as a function of energy per nucleon from Chatterjee and Magee as dashed lines while projections from the present model are shown as solid lines for ions from atomic number 1 to 100 , and for energies from 0.1 to $100 \mathrm{MeV} / \mathrm{n}$. The curves are quite similar qualitatively at energies above $1 \mathrm{MeV} / \mathrm{n}$, though there is a quantitative difference. Data

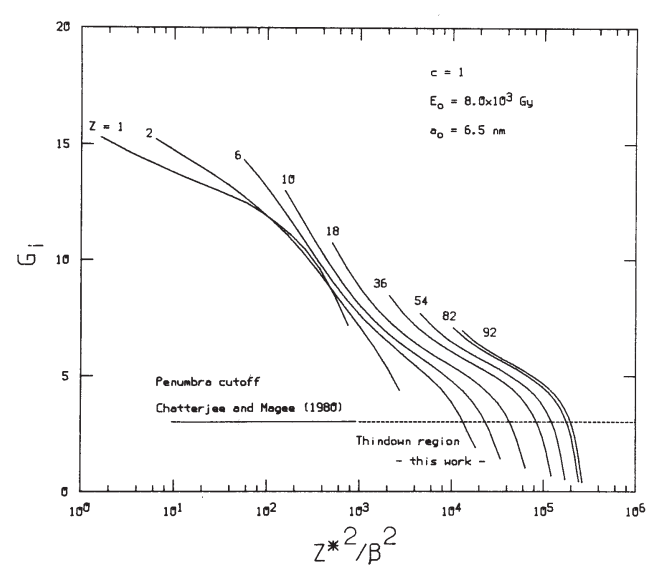

Figure 6 . The calculated action cross section, from the present model is plotted against $Z^{* 2} / \beta^{2}$, displaying the "hooks" predicted for the thindown region. This is the fundamental calculation of the present model, from which the relative effectiveness and subsequently the $G$ value are calculated.

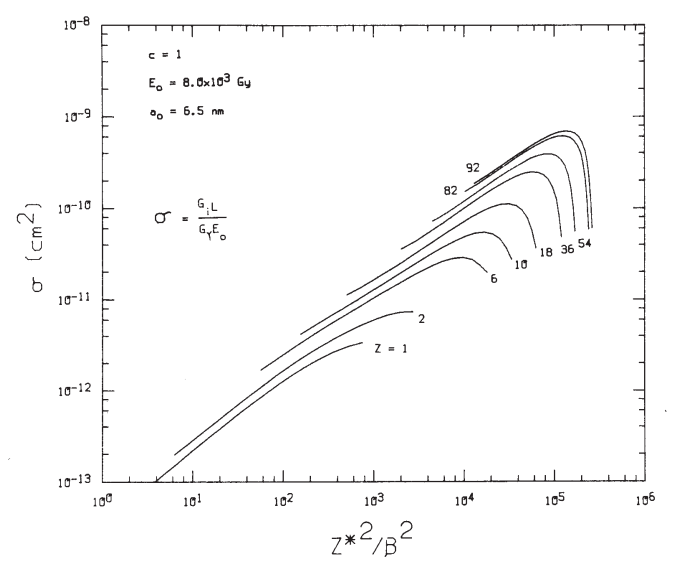

Figure 5. Calculations of the present model, from Figure 4 are here replotted to display $G_{0}$ as a function of $Z^{* 2} / \beta^{2}$ for comparison with a similar figure plotted by Chatterjee and Magee (1980). In the latter case a lower limit is imposed because of "penumbra cutoff," shown by a dashed line, which intersects the present calculation in the region of "thindown."

from a number of sources is shown superimposed upon the calculations including Hart et al. (1956), Anderson (1961), Schuler and Allen (1957), Kochanny (1963), Gordon and Hart (1961), and Schuler (1967).

In their Figure 5, Chatterjee and Magee display the relation between their calculations and experimental data. Ours, displayed here, seem somewhat closer at energies above $1 \mathrm{MeV} / \mathrm{u}$. Some of the available data, and the calculations of Chatterjee and Magee show an increase in $G$ as the energy decreases below $1 \mathrm{MeV} / \mathrm{n}$ for He and $\mathrm{H}$ ions. Our work does not enable us to make any statement about these results.

For completeness we display in Figure 5 a plot of our calculated ions yields vs $Z^{* 2} / \beta^{2}$, similar to Figure 7 of Chatterjee and Magee, except that these authors make a "penumbra cutoff" that appears at low values of $G_{i}$, where we make calculations for the "thin-down" region, attributed to the kinematic limit in delta-ray energy. Note that penumbra cutoff and thindown are not the same concept. The penumbra cutoff is associated with the radius of a "core" determined by the Bohr adiabatic limit, while the thindown is associated with the limits imposed by kinematics on the maximum energy of delta-rays, hence the maximum radial extent of the penumbra.

We display in Figure 6 calculated values of the action cross section as a function of $Z^{* 2} / \beta^{2}$, to display the hooks associated with thindown which are reflected in the lower part of Figure 5, below the dashed line.

Finally we think it is of interest to display, in Figure 7 , calculated models of the tracks of single ions in the Fricke dosimeter. These are made from the radial distribution of dose and our parameters. Each small circle represents the position of an $\mathrm{Fe}^{2+}$ ion converted to $\mathrm{Fe}^{3+}$. The picture is a slice through the medium of thickness 


\section{Fricke Dosimeter}

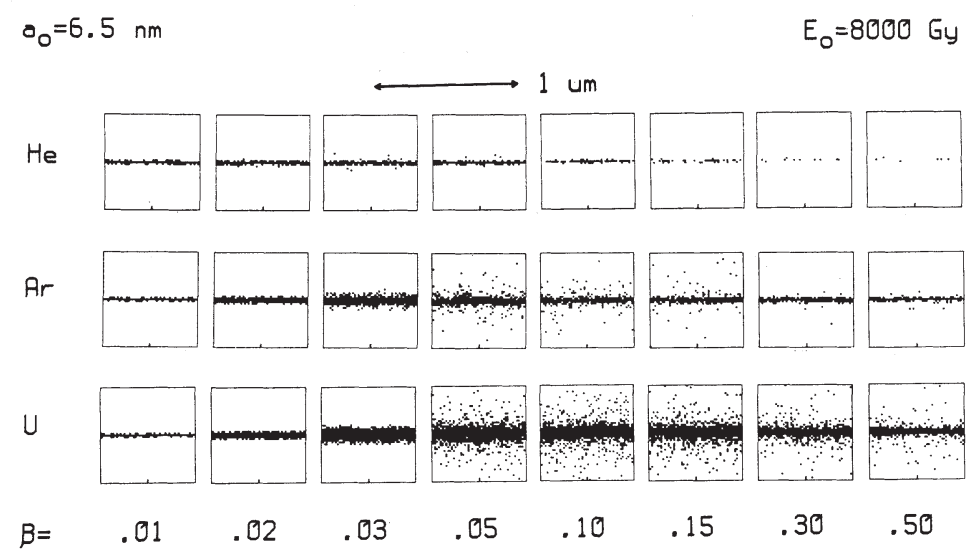

Figure 7. As in a Monte Carlo calculation, we generate particle tracks displaying the spatial distribution of $\mathrm{Fe}^{3+}$ ions after passage of the ion and the chemistry is completed, by applying random numbers to the average radial distribution of probability for activation of a sensitive volume. The simulations show the "grain count" regime in the track of a He ion at high velocities, the track width regime, and thindown, especially in the track of a U ion approaching the stopping end of its path. We display a section $13 \mathrm{~nm}$ thick (the diameter of the sensitive volume) along which the ion's path is centered.

one target diameter, and contains the same information as that represented by the calculated cross section, except that the cross section represents an average value while the track representation represents an individual track with all its fluctuations. No attempt has been made here to group interactions along individual delta-ray paths. The illustration is made from the average radial dose distribution, and therefore from the average radial probability for the oxidation of $\mathrm{Fe}^{2+}$, converted into this display by use of random numbers.

We think that these results make it evident that the Fricke dosimeter is a 1-hit detector, as we postulated in 1972. Since track theory and diffusion kinetics yield quite similar results for particles of energy above 1 $\mathrm{MeV} / \mathrm{u}$, it is likely that both models are doing the same thing, especially in the penumbral region, that is, calculating the response to a (quasi) gamma-ray irradiation of dose appropriate to the radial distance. It would be mutually advantageous if the detailed diffusion kinetic model were applied to gamma-ray or electron irradiation, in an attempt to provide an $a b$ initio explanation of the parameters of the 1-hit detector model.

\section{Acknowledgements}

We thank our colleague Prof. Dan Schlitt for his advice on software and hardware problems and Keith Williams for his preparation of Figure 7. One of the authors (MPRW) has been supported by a fellowship from the International Atomic Energy Agency (Vienna). This work has been supported by the United States Department of Energy.

\section{References}

Anderson A. R. and Hart E. J. (1961) Molecular product and free radical yields in the decomposition of water by protons, deuterons, and helium ions. Radiat. Res. 14, 689-704.

Butts J. J. and Katz R. (1967) Theory of RBE for heavy ion bom- bardment of dry enzymes and viruses. Radiat. Res. 30, 855-871.

Chatterjee A. and Magee J. L. (1980) Radiation chemistry of heavy-particle tracks -2. Fricke dosimeter system. J. Phys Chem. 84, 3537-3543.

Christman E. A., Appleby A., and Jayko M. (1981) Radiation chemistry of high-energy carbon, neon and argon ions: integral yields from ferrous sulfate solutions. Radiat. Res. 85, 443-57.

Dertinger H. and Jung H. (1970) Molecular Radiation Biology. Springer, New York.

Gordon S. and Hart E. J. (1961) Chemical yields of ionizing radiations in aqueous solutions: effect of energy of alpha particles. Radiat. Res. 15, 440-451.

Hart E. J., Ramler W. J., and Rocklin S. R. (1956) Chemical yields of ionizing particles in aqueous solutions: effect of energy of protons and deuterones. Radiat. Res. 4, 378-393.

Hart E. J. (1963) Chemical dosimetry at high dose rates, Proc. Int. Conf. on Radiation Research, pp. 39-48, U.S. Natick Laboratories.

Janni J. F. (1982) Proton range-energy tables, $1 \mathrm{KeV}-10 \mathrm{GeV}$, At. Data Nucl. Data Tables 27, 147-529.

Katz R., Sharma S. C., and Homayoonfar M. (1972) Detection of energetic heavy ions. Nucl. Instrum. Meth. 100, 13-32.

Kochanny G. L., Jr., Timnick A., Hochanadel C. J., and Goodman C. D. (1963) Radiation chemistry studies of water as related to the initial linear energy transfer of $11-\mathrm{MeV}$ to $23-$ $\mathrm{MeV}$ protons. Radiat. Res. 19, 462-473.

La Verne J. A. and Schuler R. H. (1983) Decomposition of water by very high linear energy transfer radiations. J. Phys. Chem. 87, 4564-4565.

Schuler R. H. and Allen A. O. (1957) Radiation chemistry studies with cyclotron beams of variable energy: yields in aerated ferrous sulfate solution. J. Am. Chem. Soc. 79, 1566-1572.

Schuler R. H. (1958) The effect of ferrous sulfate concentration on the yield of oxidation of ferrous ion by radiations of high LET. Radiat. Res. 8, 388-391. 
Schuler R. H. (1967) Radiation chemical studies with heavy ion radiations. J. Phys. Chem. 71, 3712-3713.

Sehested K., Bjergbakke E., Rasmussen O. L., and Fricke H. (1969) Reactions of $\mathrm{H}_{2} \mathrm{O}_{3}$ in the pulse-irradiated $\mathrm{Fe}(\mathrm{II})-\mathrm{O}_{2}$ system. Chem. Phys. 51, 3159-3166.

Waligorski M. P. R., Hamm R. N., and Katz R. (1986) The radial distribution of dose around the path of a heavy ion in liquid water. Nucl. Tracks 11, 309-319.

\section{Appendix}

\section{Radial Distribution Of Dose}

In the present work, we apply a new set of formulas for the radial distribution of dose around the path of a heavy ion, $D_{2}(t)$, elaborated elsewhere (Waligorski, 1986), as summarized below.

$$
D_{2}(t)=D_{1}(t)(1+K(t))
$$

where

(a) for $t>B=0.1 \mathrm{~nm}$ :

$$
\begin{aligned}
& K(t)=A\left(\frac{t-B}{C}\right) \exp -\left(\frac{t-B}{C}\right) \\
& B=0.1 \mathrm{~nm} \\
& C=1.5 \mathrm{~nm}+5 \mathrm{~nm} \times \beta
\end{aligned}
$$

and

or

$$
A=8 \beta^{1 / 3} \text { for } \beta<0.03
$$

$$
A=19 \beta^{1 / 3} \text { for } \beta>0.03
$$

(b) for $t<B=0.1 \mathrm{~nm}$

$$
K(t)=0
$$

and

$$
D_{1}(t)=\frac{N e^{4} Z^{* 2}}{\alpha m c^{2} \beta^{2} t}\left[\frac{\left(1-\frac{t+\theta}{T+\theta}\right)^{1 / \alpha}}{t+\theta}\right]
$$

where $D_{2}(t)$ is the dose deposited in a coaxial cylindrical shell of thickness $\mathrm{d} t$ at a distance $t$ from the path of an ion of effective charge $Z^{*}$ moving with a relative velocity $\beta=v / c$ ( $c$ is the speed of light) through the detector medium containing $N$ electrons per $\mathrm{cm}^{3}, m$ is the mass of the electron. The Rutherford cross-section for delta-ray production from atoms having ionization potential $I=10 \mathrm{eV}$, normal ejection and a power law range $(r)$-energy $(w)$ relationship for electrons, are assumed. The range-energy relationship is based on a twocomponent fit to the available experimental data concerning ranges of electrons in aluminum:

$$
r=k w^{\alpha}
$$

where

$$
k=6 \times 10^{-6} \mathrm{~g} \mathrm{~cm}^{-2} \mathrm{keV}^{-\alpha}
$$

For

$$
w<1 \mathrm{keV}, \quad \alpha=1.079 ; \quad \text { for } w>1 \mathrm{keV}, \quad \alpha=1.667
$$

$\theta$ is the "range" of an electron of energy $w=I$; that is

$$
\theta=k(0.010 \mathrm{keV})^{1.079}=4.17 \times 10^{-8} \mathrm{~g} \mathrm{~cm}^{-2} .
$$

The kinematically limited maximum delta-ray energy is:

$$
W=2 m c^{2} \beta^{2} /\left(1-\beta^{2}\right) \text {. }
$$

This translates to the maximum range of delta-rays:

$$
T=k W^{\alpha}
$$

where the choice of $\alpha$ depends on the relative velocity $\beta$ of the ion. We calculate:

$$
\begin{aligned}
& \text { for } \beta<0.03, \alpha=1.079, \\
& \text { and for } \beta>0.03, \alpha=1.667 .
\end{aligned}
$$

For water

$$
2 \pi \mathrm{Ne}^{4} / m c^{2}=1.369 \times 10^{-7} \mathrm{erg} / \mathrm{cm}=8.5 \mathrm{keV} \mathrm{mm}^{-1} .
$$

The effective charge number of an ion of atomic number $Z$ moving with relative speed $\beta$ is

$$
Z^{*}=Z\left[1-\exp \left(-125 \beta Z^{-2 / 3}\right)\right] .
$$

In the preceding formulas the expression $D_{1}(t)$ was calculated from the Rutherford formula, and includes only half the energy deposited by the ion. Provisionally we think of it as the energy deposited by the delta-rays. The contribution $K(t) D_{1}(t)$ is generated from a Monte Carlo calculation of the radial dose distribution in liquid water, and provisionally is thought to represent the excitation energy contributed by the primary ion. Together, as in equation (A.1) these integrate radially to give the stopping power of a proton in liquid water to within $10 \%$ over a wide range in proton speeds. The contribution from $K(t)$ principally appears as a "hump" in a plot of the radial dose distribution at radial distances 1-10 nm.

We find that the contribution of the primary energy to the action cross section of a 1-hit detector varies with its radiosensitivity and target size, and with the charge and speed of the bombarding ion. Essentially it can be expected to be more important in the grain count regime, where the track can be thought of as a "string of randomly separated beads," and diminishes in importance in the track width regime, where the track is like a "hairy rope," and there is much overkill in the innermost $10 \mathrm{~nm}$ from the ion's path.

The "extended target" calculation now proceeds as follows: We calculate the average dose distribution, $E\left(z, \beta, t, a_{0}\right)$ in a sensitive element of radius $a_{0}$, represented by a chunky cylinder of this radius, the axis of which lies at the distance $t$ from the ion's path, by integrating over its volume the appropriate formula for the radial distribution of dose and proceed to calculate the radial distribution of probability and thence the action cross section. 